\title{
Effect of Human Mobility On The Spatial Spread of Airborne Diseases: An Epidemic Model With Indirect Transmission
}

Jummy Funke David ( $\square$ jummy30@yorku.ca )

York University https://orcid.org/0000-0002-5995-3303

Sarafa A. lyaniwura

The University of British Columbia

\section{Research Article}

Keywords: Human mobility, heterogeneous mixing, epidemics, airborne disease, indirect transmission, asymptotic analysis, Green's function.

Posted Date: October 26th, 2021

DOI: https://doi.org/10.21203/rs.3.rs-965234/v1

License: (c) (i) This work is licensed under a Creative Commons Attribution 4.0 International License.

Read Full License 


\title{
Effect of human mobility on the spatial spread of airborne diseases: an epidemic model with indirect transmission
}

\author{
Jummy F. David ${ }^{1^{*}}$ and Sarafa A. Iyaniwura ${ }^{2 *}$
}

1 Department of Mathematics and Statistics, York University, Toronto, Ontario, Canada. 2 Department of Mathematics and Institute of Applied Mathematics, University of British Columbia, Vancouver, BC, Canada.

*Correspondence: jummy30@yorku.ca \& iyaniwura@math.ubc.ca

\begin{abstract}
We extended a class of coupled PDE-ODE models for studying the spatial spread of airborne diseases by incorporating human mobility. Human populations are modeled with patches, and a Lagrangian perspective is used to keep track of individuals' places of residence. The movement of pathogens in the air is modeled with linear diffusion and coupled to the SIR dynamics of each human population through an integral of the density of pathogen around the population patch. In the limit of fast diffusion pathogens, the method of matched asymptotic analysis is used to reduce the coupled PDE-ODE model to a nonlinear system of ODEs for the average density of pathogens in the air. The reduced system of ODEs is used to derive the basic reproduction number and the final size relation for the model. Numerical simulations of the full PDE-ODE model and the reduced system of ODEs are used to assess the impact of human mobility, together with the diffusion of pathogens on the dynamics of the disease. Results from the two models are consistent and show that human mobility significantly affects disease dynamics. In addition, we show that an increase in the diffusion rate of pathogen leads to a smaller epidemic.
\end{abstract}

Keywords: Human mobility, heterogeneous mixing, epidemics, airborne disease, indirect transmission, asymptotic analysis, Green's function.

\section{Introduction}

Many airborne diseases are transmitted directly via host-host and/or indirectly through the host-source-host [3, 18]. In the context of models for infectious diseases transmission, Lagrangian approach offers a theoretical framework that explicitly tracks the heterogeneity of host population and mobility [12]. Despite the complexity of explicitly considering the mixing patterns and connections that exist between local communities and different regions, Lagrangian method used for residence time has shown its ability to analytically explain the failure of feasible mobility restriction measures and their impact on disease transmission [6,12]. Several mathematical models that studied heterogeneity and different mobility scenarios have been explored within the heterogeneous mixing framework [5, 6, 12], however many of these studies have considered epidemic models with no diffusion of pathogens and/or indirect transmission route.

Considering a more realistic scenario (in the case of superspreaders), but simpler than the detailed networks or PDE models, could be a possibility. To model the effect of human mobility, with heterogeneous mixing, one may assume that the population is divided into subgroups with different activity levels. In this study, we extend the novel coupled PDE-ODE model of [14] used to study the spatial spread of airborne diseases by incorporate human mobility. We base our analytical results on the reproduction number and the final epidemic size in an heterogeneous mixing environment. 
Our goal is to study the effect of human mobility and heterogeneous mixing on the dynamics of the disease. We use the Lagrangian method to track an individual's place of residence at all time following the approach in $[2,5,12,13$, $17,15]$. This allows us to theoretically and numerically show how humans moving between patches affect the spread of the diseases. Our extended model which includes human mobility and a diffusion term, may be an alternative way to study the spread of airborne diseases in an heterogeneous mixing environment.

The rest of the paper is structured as follows. In Section 2, we extend the coupled PDE-ODE model developed in [14] by incorporating human mobility using the Lagrangian method. We non-dimensionalize the extended model in Section 2.1, and used matched asymptotic expansions method to reduce the dimensionless coupled model to a nonlinear system of ODEs, in the limit of fast diffusing pathogens. In Section 3.1 and 3.2, for a scenario with two populations, the reduced ODE model is used to compute the basic reproduction number and final size relation of the epidemic, respectively. The coupled PDE-ODE model together with the reduced system of ODEs are used to numerically study the effect of different human mobility patterns on the disease dynamics for a two population patch scenario. A brief discussion of results and possible future directions concludes the paper in Section 4.

\section{Mathematical model}

The novel coupled PDE-ODE model of [14], used to study the spatial spread of airborne diseases between human populations, is extended to incorporate human movement between patches. The heterogeneous mixing model keeps track of individuals' place of residence over time. The model is non-dimensionalized and studied in the limit of fast diffusion pathogens. In this limit, we use the method of matched asymptotic analysis to reduce the dimensionless coupled PDE-ODE model to a nonlinear system of ODEs for the average density of pathogens in the environment. The reduced system of ODEs is then used to compute the basic reproduction number and final size relation for a scenario with two population patches.

We represent human populations with localized patches that have partially transmitting boundaries through which pathogens are shed into the atmosphere by infected individuals. These pathogens diffuse and decay at a constant rate in the air. A susceptible individual becomes infected by coming in contact with pathogens (indirect transmission pathway). We assume that the spread of infection in each population patch depends on the density of pathogens around the patch, and did not explicitly model pathogens within the patches. Similarly, we model human movement between patches using a Lagrangian method. Let $\Omega \subset \mathbb{R}^{2}$ be our 2-D bounded domain of interest containing $m$ population patches represented by $\Omega_{j}$ for $j=1, \ldots, m$, which are separated by an $O(1)$ distance. In the region between the patches $\Omega \backslash \cup_{j=1}^{m} \Omega_{j}$ (bulk region), the density of pathogens $\mathcal{P}(\boldsymbol{X}, T)$ satisfies

$$
\begin{gathered}
\frac{\partial \mathcal{P}}{\partial T}=D_{B} \Delta \mathcal{P}-\delta \mathcal{P}, \quad T>0, \quad \boldsymbol{X} \in \Omega \backslash \cup_{j=1}^{m} \Omega_{j} ; \\
\partial_{n_{X}} \mathcal{P}=0, \quad \boldsymbol{X} \in \partial \Omega ; \quad D_{B} \partial_{n_{X j}} \mathcal{P}=-r_{j} \mathcal{I}_{j}, \quad \boldsymbol{X} \in \partial \Omega_{j}, \quad j=1, \ldots, m,
\end{gathered}
$$

where $D_{B}>0$ and $\delta>0$ respectively represent the dimensional diffusion coefficient and decay rate of pathogens in the bulk region, $r_{j}>0$ is the dimensional shedding rate of pathogen by an infected individual in the $j^{\text {th }}$ patch, and $\partial_{n_{X}}$ is the outer normal derivative on the boundary of the patches pointing into the bulk region. The dynamics of the diffusing pathogens in the bulk region is coupled to the population dynamics of the $j^{\text {th }}$ patch as follows

$$
\begin{aligned}
& \frac{d \mathcal{S}_{j}}{d T}=-\sum_{i=1}^{m} \mu_{i} \mathcal{S}_{j} \gamma_{j i} \int_{\partial \Omega_{i}}\left(\mathcal{P} / p_{c}\right) \mathrm{d} S_{\boldsymbol{X} i} ; \\
& \frac{d \mathcal{I}_{j}}{d T}=\sum_{i=1}^{m} \mu_{i} \mathcal{S}_{j} \gamma_{j i} \int_{\partial \Omega_{i}}\left(\mathcal{P} / p_{c}\right) \mathrm{d} S_{\boldsymbol{X} i}-\alpha_{j} \mathcal{I}_{j} ; \\
& \frac{d \mathcal{R}_{j}}{d T}=\alpha_{j} \mathcal{I}_{j}, \quad j=1, \ldots, m,
\end{aligned}
$$

where $\mathcal{S}_{j}, \mathcal{I}_{j}$, and $\mathcal{R}_{j}$ are the susceptible, infected, and recovered/removed population in the $j^{\text {th }}$ patch, respectively, with the total population at time $T$ written as $\mathcal{N}_{j}(T)=\mathcal{S}_{j}(T)+\mathcal{I}_{j}(T)+\mathcal{R}_{j}(T)$. We assume that the population of the entire system is constant throughout the epidemic period, that is, $N=\sum_{j=1}^{m} N_{j}(t)$. Here, $\gamma_{j i}$ is fraction of the residence 
of patch $j$ that are in patch $i$, where $\sum_{i=1}^{m} \gamma_{j i}=1$ for $j=1, \ldots, m$. The parameters $\mu_{j}$ and $\alpha_{j}$ are the dimensional transmission and recovery rates, respectively, for individuals in the $j^{\text {th }}$ patch, and $p_{c}$ is a typical value for the density of pathogens. The integrals in (2.1c) and (2.1d) are over the boundary of the $j^{\text {th }}$ patch and are used to account for the total density of pathogens around the patch. These terms show that the spread of infection within a patch depends on the density of pathogens around the patch. It is important to emphasize that our model does not explicitly account for pathogens within the patches. The Robin boundary condition $D_{B} \partial_{n_{X}} \mathcal{P}=-r_{j} \mathcal{I}_{j}$ on the boundary of the $j^{\text {th }}$ patch accounts for the amount of pathogens shed into the atmosphere by infected individuals in the patch. This condition shows that the amount of pathogens shed into the atmosphere from the $j^{\text {th }}$ patch depends on the population of infected individuals in the patch.

\subsection{Non-dimensionalization of the coupled PDE-ODE model}

Next, we non-dimensionalize the coupled PDE-ODE model (2.1). The dimensions of the variables and parameters of the model are defined as follow:

$$
\begin{aligned}
& {[\mathcal{P}]=\frac{\text { pathogens }}{(\text { length })^{2}}, \quad\left[D_{B}\right]=\frac{(\text { length })^{2}}{\text { time }}, \quad\left[p_{c}\right]=\text { pathogens }, \quad[T]=\text { time },} \\
& {[X]=\text { length }, \quad\left[\mu_{j}\right]=\frac{\text { length }}{\text { time }}, \quad\left[\mathcal{N}_{j}\right]=\left[\mathcal{S}_{j}\right]=\left[\mathcal{I}_{j}\right]=\left[\mathcal{R}_{j}\right]=\text { individuals },} \\
& {\left[r_{j}\right]=\frac{\text { pathogens }}{\text { individual } \times \text { time } \times \text { length }}, \quad[\delta]=\left[\alpha_{j}\right]=\frac{1}{\text { time }}, \quad i, j=1, \ldots, m .}
\end{aligned}
$$

where [ $\Upsilon$ ] represents the dimension of $\Upsilon$. We assume that the population patches are circular with common radius $\rho$, which is small compared to the length-scale $L$ of the domain $\Omega$, and introduce a small scaling parameter $\varepsilon=\rho / L \ll 1$. The dimensionless variables are defined as follows

$$
P=\frac{L^{2}}{p_{c}} \mathcal{P}, \quad S_{j}=\frac{\mathcal{S}_{j}}{\mathcal{N}_{j}}, \quad I_{j}=\frac{\mathcal{I}_{j}}{\mathcal{N}_{j}}, \quad R_{j}=\frac{\mathcal{R}_{j}}{\mathcal{N}_{j}}, \quad x=\frac{X}{L}, \quad t=\delta T .
$$

So that $S_{j}, I_{j}$, and $R_{j}$ are the proportion of susceptible, infected, and recovered/removed individuals in the $j^{\text {th }}$ patch, respectively, and $P \equiv P(\boldsymbol{x}, t)$ is the dimensionless density of pathogens in the bulk region. Upon substituting (2.3) into (2.1), we derive that $P(\boldsymbol{x}, t)$ satisfies

$$
\begin{gathered}
\frac{\partial P}{\partial t}=D \Delta P-P, \quad t>0, \quad x \in \Omega \backslash \cup_{j=1}^{m} \Omega_{\varepsilon j} ; \\
\partial_{n_{x}} P=0, \quad x \in \partial \Omega ; \quad D \partial_{n_{x}} P=-r_{j}\left(\frac{\mathcal{N}_{j} L}{\delta p_{c}}\right) I_{j}, \quad x \in \partial \Omega_{\varepsilon j}, \quad j=1, \ldots, m,
\end{gathered}
$$

where $D \equiv D_{B} /\left(\delta L^{2}\right)$ is the effective diffusion rate of pathogens in the bulk region. From the ODE system $((2.1 \mathrm{c})$ (2.1e)), we derive the dimensionless system of ODEs for the population dynamics of the $j^{\text {th }}$ patch as

$$
\begin{aligned}
\frac{d S_{j}}{d t} & =-\sum_{i=1}^{m}\left(\frac{\mu_{i}}{\delta L}\right) S_{j} \gamma_{j i} \int_{\partial \Omega_{\varepsilon i}} P \mathrm{~d} s_{x} \\
\frac{d I_{j}}{d t} & =\sum_{i=1}^{m}\left(\frac{\mu_{i}}{\delta L}\right) S_{j} \gamma_{j i} \int_{\partial \Omega_{\varepsilon i}} P \mathrm{~d} s_{x}-\phi_{j} I_{j} \\
\frac{d R_{j}}{d t} & =\phi_{j} I_{j}, \quad j=1, \ldots, m,
\end{aligned}
$$

where $\phi_{j}=\alpha_{j} / \delta$ is the dimensionless recovery rate and $\Omega_{\varepsilon j}=\left\{\boldsymbol{x}:\left|\boldsymbol{x}_{j}-\boldsymbol{x}\right|<\varepsilon\right\}$ represents the $j^{\text {th }}$ patch of radius $\varepsilon \ll 1$ with center at $\boldsymbol{x}_{j}$. Note that we have used the scaling $\mathrm{d} S_{\boldsymbol{X}}=L \mathrm{~d} s_{\boldsymbol{x}}$ in the integrals on the boundary of the patches. We set

$$
\frac{\beta_{j}}{2 \pi \varepsilon}=\frac{\mu_{j}}{\delta L} \quad \text { and } \quad \frac{\sigma_{j}}{2 \pi \varepsilon}=r_{j} \frac{\mathcal{N}_{j} L}{\delta p_{c}}
$$


where $\beta_{j}$ and $\sigma_{j}$ are $O(1)$. We have assumed that $\left(\mu_{j} / \delta L\right)$ and $r_{j}\left(\mathcal{N}_{j} L / \delta p_{c}\right)$ are $O(1 / \varepsilon)$ in order to effectively capture the density of pathogens shed into the bulk region by infected individuals in each patch, since the patches are relatively small compared to the length-scale of the domain. This re-scaling enables us to write the dimensionless transmission and shedding rates, $\beta_{j}$ and $\sigma_{j}$, respectively, as functions of the circumference of the $j^{\text {th }}$ patch. Upon substituting (2.6) into (2.4) and (2.5), we obtain that the dimensionless density of the pathogens $P(\boldsymbol{x}, t)$ satisfies

$$
\begin{gathered}
\frac{\partial P}{\partial t}=D \Delta P-P, \quad t>0, \quad x \in \Omega \backslash \cup_{j=1}^{m} \Omega_{\varepsilon j} ; \\
\partial_{n_{x}} P=0, \quad x \in \partial \Omega ; \quad 2 \pi \varepsilon D \partial_{n_{x}} P=-\sigma_{j} I_{j}, \quad x \in \partial \Omega_{\varepsilon j}, \quad j=1, \ldots, m,
\end{gathered}
$$

which is coupled to the dimensionless $S I R$ dynamics of the $j^{\text {th }}$ patch as follows

$$
\begin{aligned}
\frac{d S_{j}}{d t} & =-\frac{1}{2 \pi \varepsilon} \sum_{i=1}^{m} \beta_{i} S_{j} \gamma_{j i} \int_{\partial \Omega_{\varepsilon j}} P \mathrm{~d} s_{\boldsymbol{x}} ; \\
\frac{d I_{j}}{d t} & =\frac{1}{2 \pi \varepsilon} \sum_{i=1}^{m} \beta_{i} S_{j} \gamma_{j i} \int_{\partial \Omega_{\varepsilon j}} P \mathrm{~d} s_{\boldsymbol{x}}-\phi_{j} I_{j} ; \\
\frac{d R_{j}}{d t} & =\phi_{j} I_{j}, \quad j=1, \ldots, m,
\end{aligned}
$$

where

$$
\beta_{j}=\frac{2 \pi \varepsilon}{\delta L} \mu_{j}, \quad \sigma_{j}=\frac{2 \pi \varepsilon}{\delta p_{c}} r_{j} \mathcal{N}_{j} L \quad \text { and } \quad \phi_{j}=\frac{\alpha_{j}}{\delta} .
$$

are the dimensionless transmission, shedding and recovery rates for the $j^{\text {th }}$ patch, respectively.

Table 1: Model parameters, descriptions, and values.

\begin{tabular}{clrr}
\hline Parameter & Description & Patch 1, 2 values & Reference \\
\hline$\mu$ & Dimensional effective contact rate & $0.3,1.2$ & {$[2]$} \\
$\beta$ & Dimensionless effective contact rate & Computed using (2.8) & Derived \\
$r$ & Dimensional pathogen shedding rate & $0.1,1$ & {$[22]$} \\
$\sigma$ & Dimensionless pathogen shedding rate & Computed using (2.8) & Derived \\
$\alpha$ & Dimensional recovery rate & 1.87 & {$[22]$} \\
$\phi$ & Dimensionless recovery rate & Computed using (2.8) & Derived \\
$N_{1}, N_{2}$ & Total population & 300,250 & Assumed \\
$D_{B}$ & Dimensional diffusion rate of pathogens & & \\
$D$ & Dimensionless diffusion rate of pathogens & Varied & \\
$D_{0}$ & Scaled dimensionless diffusion rate of pathogens & Computed using (2.9) & Derived \\
$\gamma_{11}$ & Fraction of patch 1 residence currently in patch 1 & Varied & \\
$\gamma_{12}$ & Fraction of patch 1 residence currently in patch 2 & Varied & \\
$\gamma_{21}$ & Fraction of patch 2 residence currently in patch 1 & Varied & \\
$\gamma_{22}$ & Fraction of patch 2 residence currently in patch 2 & Varied & \\
$\delta$ & Dimensionless decay rate of pathogens & 0.25 & Assumed \\
$p_{c}$ & Typical value for density of pathogens & 0.01 & Assumed \\
$\varepsilon$ & Radius of the population patch & 0.02 & Assumed \\
$\Omega$ & Area of the domain (unit disk) & $\pi$ & Derived \\
\hline
\end{tabular}

Table 1 shows all parameters and their respective descriptions.

Next, we study the dimensionless coupled model (2.7) in the limit of fast diffusing pathogens, where $D=O\left(v^{-1}\right) \gg 1$, with $v=-1 / \log _{e}(\varepsilon)$ and $\varepsilon \ll 1$, using the method of matched asymptotic expansions. We define

$$
D=\frac{D_{0}}{v}, \quad \text { where } \quad D_{0}=O(1) \quad \text { and } \quad v=-\frac{1}{\log _{e}(\varepsilon)} \ll 1
$$


Substituting $D=D_{0} / v$ into (2.7a) and (2.7b), we obtain

$$
\begin{gathered}
\frac{\partial P}{\partial t}=\frac{D_{0}}{v} \Delta P-P, \quad t>0, \quad x \in \Omega \backslash \cup_{j=1}^{m} \Omega_{\varepsilon j} ; \\
\partial_{n_{x}} P=0, \quad x \in \partial \Omega ; \quad 2 \pi \varepsilon \frac{D_{0}}{v} \partial_{n_{x}} P=-\sigma_{j} I_{j}, \quad x \in \partial \Omega_{\varepsilon j}, \quad j=1, \ldots, m,
\end{gathered}
$$

Following the approach of [14], we derive a two-term asymptotic expansion in terms of $v$ for the density of pathogens in the bulk region given by

$$
P=P_{0}+\frac{v}{D_{0}} \sum_{i=1}^{m} \sigma_{i} I_{i} G\left(\boldsymbol{x} ; \boldsymbol{x}_{i}\right)+\ldots,
$$

where $P_{0} \equiv P_{0}(t)$ is the average density of pathogens in the bulk region which satisfies

$$
\frac{d P_{0}}{d t}=-P_{0}+\frac{1}{|\Omega|} \sum_{j=1}^{m} \sigma_{j} I_{j}
$$

and $G\left(\boldsymbol{x} ; \boldsymbol{x}_{j}\right)$ is the Neumann's Green function satisfying

$$
\begin{gathered}
\Delta G=\frac{1}{|\Omega|}-\delta\left(x-x_{j}\right), \quad x \in \Omega ; \quad \partial_{n} G=0, \quad x \in \partial \Omega ; \\
G\left(x ; x_{j}\right) \sim-\frac{1}{2 \pi} \log _{e}\left|x-x_{j}\right|+\Re_{j}, \quad \text { as } \quad x \rightarrow x_{j}, \quad \text { and } \quad \int_{\Omega} G \mathrm{~d} x=0,
\end{gathered}
$$

with regular part $\Re_{j} \equiv \Re\left(\boldsymbol{x}_{j}\right)$. Similarly, near the $j^{\text {th }}$ population patch, we derive

$$
Q_{j}=\left(P_{0}(t)+\frac{\sigma_{j} I_{j}}{2 \pi D_{0}}\right)+\frac{v}{D_{0}}\left[-\left(\frac{\sigma_{j} I_{j}}{2 \pi}\right) \log _{e}\left(\frac{\left|\boldsymbol{x}-\boldsymbol{x}_{j}\right|}{\varepsilon}\right)+\sigma_{j} I_{j} \Re_{j}+\sum_{i \neq j}^{m} \sigma_{i} I_{i} G\left(\boldsymbol{x}_{j} ; \boldsymbol{x}_{i}\right)\right]+\ldots, \quad j=1, \ldots, m,
$$

On the boundary of the $j^{\text {th }}$ patch, where $\left|\boldsymbol{x}-\boldsymbol{x}_{j}\right|=\varepsilon$, (2.14) reduces to

$$
Q_{j}=\left(P_{0}(t)+\frac{\sigma_{j} I_{j}}{2 \pi D_{0}}\right)+\frac{v}{D_{0}}\left(\sigma_{j} I_{j} \Re_{j}+\sum_{i \neq j}^{m} \sigma_{i} I_{i} G\left(\boldsymbol{x}_{j} ; \boldsymbol{x}_{i}\right)\right)+\ldots, \quad j=1, \ldots, m,
$$

To couple the dynamics of the diffusing pathogens to that of human population in the $j^{\text {th }}$ patch, we substitute (2.15) into the ODE system (2.7c) to obtain

$$
\begin{aligned}
\frac{d S_{j}}{d t} & =-\sum_{i=1}^{m} \beta_{i} S_{j} \gamma_{j i}\left(p(t)+\frac{\sigma_{i} I_{i}}{2 \pi D_{0}}\right)-\frac{v}{D_{0}} \sum_{i=1}^{m} \beta_{i} S_{j} \gamma_{j i} \Psi_{i} \\
\frac{d I_{j}}{d t} & =\sum_{i=1}^{m} \beta_{i} S_{j} \gamma_{j i}\left(p(t)+\frac{\sigma_{i} I_{i}}{2 \pi D_{0}}\right)-\frac{v}{D_{0}} \sum_{i=1}^{m} \beta_{i} S_{j} \gamma_{j i} \Psi_{i}-\phi_{j} I_{j} \\
\frac{d R_{j}}{d t} & =\phi_{j} I_{j}, \quad j=1, \ldots, m,
\end{aligned}
$$

where $\Psi_{j}=(\mathcal{G} \Phi)_{j}$ is the $j^{\text {th }}$ entry of the vector $\mathcal{G} \Phi$, with $\Phi=\left(\sigma_{1} I_{1}, \ldots, \sigma_{m} I_{m}\right)^{T}$. Here, $\mathcal{G}$ is the Neumann Green's matrix whose entries are defined by

$$
(\mathcal{G})_{j j}=\Re\left(x_{j}\right) \text { for } \quad i=j \quad \text { and } \quad(\mathcal{G})_{i j}=(\mathcal{G})_{j i}=G\left(x_{i} ; \boldsymbol{x}_{j}\right) \text { for } i \neq j,
$$

where $G\left(\boldsymbol{x}_{j} ; \boldsymbol{x}_{i}\right)$ is the Neumann Green's function satisfying (2.13) and $\Re_{j} \equiv \Re\left(\boldsymbol{x}_{j}\right)$ is its regular part at the point $\boldsymbol{x}=\boldsymbol{x}_{j}$. For convenience of notation, we have replaced $P_{0}(t)$ with $p(t)$ in (2.16). Combining (2.12) and (2.16), we 
obtain an ODE systems for the average density of pathogen in the atmosphere coupled to the population dynamics in the patches that is valid in the limit $D=O\left(v^{-1}\right)$, where $v=-1 / \log (\varepsilon)$ with $\varepsilon \ll 1$. This ODE system is given by

$$
\begin{aligned}
\frac{d p(t)}{d t} & =-p(t)+\frac{1}{|\Omega|} \sum_{j=1}^{m} \sigma_{j} I_{j}, \\
\frac{d S_{j}}{d t} & =-\sum_{i=1}^{m} \beta_{i} S_{j} \gamma_{j i}\left(p(t)+\frac{\sigma_{i} I_{i}}{2 \pi D_{0}}\right)-\frac{v}{D_{0}} \sum_{i=1}^{m} \beta_{i} S_{j} \gamma_{j i} \Psi_{i}, \\
\frac{d I_{j}}{d t} & =\sum_{i=1}^{m} \beta_{i} S_{j} \gamma_{j i}\left(p(t)+\frac{\sigma_{i} I_{i}}{2 \pi D_{0}}\right)-\frac{v}{D_{0}} \sum_{i=1}^{m} \beta_{i} S_{j} \gamma_{j i} \Psi_{i}-\phi_{j} I_{j}, \\
\frac{d R_{j}}{d t} & =\phi_{j} I_{j}, \quad j=1, \ldots, m .
\end{aligned}
$$

Note that the terms with $\sigma_{j} I_{j} /\left(2 \pi D_{0}\right)$ in (2.18) do not model direct transmission, but rather they account for the pathogens shed by infected individuals in the $j^{\text {th }}$ patch. The density of these pathogens depend on the scaled-diffusion rate $D_{0}$ and the population of infected individuals in the patch. When the pathogens diffuse slowly (small $D_{0}$ ), there is significant contribution from this term, and this contribution decreases as $D_{0}$ increasing. In the limit $D_{0} \rightarrow \infty$, these terms go to zero and the ODE system reduces to the model for well-mixed regime present in equation 5 of [15]. In Sections 3, we study the reduced ODE system (2.18) for two population patches, and compute the basic reproduction number and final size relation for this scenario.

\section{Two-patch model with heterogeneous mixing}

In this section, we consider a scenario with two population patches $(m=2)$ centered at $\boldsymbol{x}_{1}=(0.5,0)$ and $\boldsymbol{x}_{2}=(-0.5,0)$ in the unit disk and use the dimensionless coupled PDE-ODE model (2.7) and the reduced system of ODEs (2.18) to study the dynamics of the disease in these populations. For this scenario, we derive from (2.7) that the dimensionless density of pathogens $P(\boldsymbol{x}, t)$ in the atmosphere satisfies

$$
\begin{gathered}
\frac{\partial P}{\partial t}=D \Delta P-P, \quad t>0, \quad x \in \Omega \backslash\left\{\Omega_{1} \cup \Omega_{2}\right\} ; \\
\partial_{n} P=0, \quad x \in \partial \Omega ; \quad 2 \pi \varepsilon D \partial_{n} P=-\sigma_{1} I_{1}, \quad x \in \partial \Omega_{1} ; \quad 2 \pi \varepsilon D \partial_{n} P=-\sigma_{2} I_{2}, \quad x \in \partial \Omega_{2},
\end{gathered}
$$

where $\Omega_{1}$ and $\Omega_{2}$ are the two population patches centered at $\boldsymbol{x}_{1}=(0.5,0)$ and $\boldsymbol{x}_{2}=(-0.5,0)$, respectively. The dynamics of the diffusion pathogens is coupled to the population dynamics of the two patches as follows

\section{Patch 1}

$$
\begin{aligned}
\frac{d S_{1}}{d t} & =-\frac{1}{2 \pi \varepsilon} \sum_{i=1}^{2} \beta_{i} S_{1} \gamma_{1 i} \int_{\partial \Omega_{\varepsilon_{i}}} P \mathrm{~d} s_{\boldsymbol{x}} \\
\frac{d I_{1}}{d t} & =\frac{1}{2 \pi \varepsilon} \sum_{i=1}^{2} \beta_{i} S_{1} \gamma_{1 i} \int_{\partial \Omega_{\varepsilon_{i}}} P \mathrm{~d} s_{\boldsymbol{x}}-\phi_{1} I_{1} \\
\frac{d R_{1}}{d t} & =\phi_{1} I_{1}
\end{aligned}
$$

Patch 2

$$
\begin{aligned}
\frac{d S_{2}}{d t} & =-\frac{1}{2 \pi \varepsilon} \sum_{i=1}^{2} \beta_{i} S_{2} \gamma_{2 i} \int_{\partial \Omega_{\varepsilon_{i}}} P \mathrm{~d} s_{\boldsymbol{x}} ; \\
\frac{d I_{2}}{d t} & =\frac{1}{2 \pi \varepsilon} \sum_{i=1}^{2} \beta_{i} S_{2} \gamma_{2 i} \int_{\partial \Omega_{\varepsilon_{i}}} P \mathrm{~d} s_{\boldsymbol{x}}-\phi_{2} I_{2} ; \\
\frac{d R_{2}}{d t} & =\phi_{2} I_{2} .
\end{aligned}
$$

Similarly, we derive the reduced ODE system for the two population patch scenario from (2.18) as

$$
\frac{d p}{d t}=-p+\frac{1}{|\Omega|}\left(\sigma_{1} I_{1}+\sigma_{2} I_{2}\right)
$$




\section{Patch 1}

$$
\begin{aligned}
\frac{d S_{1}}{d t} & =-\sum_{i=1}^{2} \beta_{i} S_{1} \gamma_{1 i}\left(p(t)+\frac{\sigma_{i} I_{i}}{2 \pi D_{0}}\right), \\
\frac{d I_{1}}{d t} & =\sum_{i=1}^{2} \beta_{i} S_{1} \gamma_{1 i}\left(p(t)+\frac{\sigma_{i} I_{i}}{2 \pi D_{0}}\right)-\phi_{1} I_{1}, \\
\frac{d R_{1}}{d t} & =\phi_{1} I_{1},
\end{aligned}
$$

Patch 2

$$
\begin{aligned}
\frac{d S_{2}}{d t} & =-\sum_{i=1}^{2} \beta_{i} S_{2} \gamma_{2 i}\left(p(t)+\frac{\sigma_{i} I_{i}}{2 \pi D_{0}}\right), \\
\frac{d I_{2}}{d t} & =\sum_{i=1}^{2} \beta_{i} S_{2} \gamma_{2 i}\left(p(t)+\frac{\sigma_{i} I_{i}}{2 \pi D_{0}}\right)-\phi_{2} I_{2}, \\
\frac{d R_{2}}{d t} & =\phi_{2} I_{2},
\end{aligned}
$$

where $p \equiv p(t)$ is the average density of pathogens in the atmosphere and $D_{0}$ is the scaled diffusion coefficient of the pathogens. The ODE system (3.2) has a similar structure to those studied in [14, 15]. The coupled PDE-ODE model (3.1) and the reduced ODE system (3.2) will be used to study the transmission dynamics of the disease for two populations scenario.

\subsection{Basic reproduction number}

Here, we use the reduced system of ODEs (3.2) to compute the basic reproduction number, $\mathcal{R}_{0}$ (the secondary infections caused by a single infective into a totally susceptible population). To use the next generational matrix approach for computing $\mathcal{R}_{0}$ as was done in [16, 19, 21], we construct a system of equations for the infectious classes given by

$$
\begin{aligned}
\frac{d I_{1}}{d t} & =\beta_{1} S_{1} \gamma_{11}\left(p(t)+\frac{\sigma_{1} I_{1}}{2 \pi D_{0}}\right)+\beta_{2} S_{1} \gamma_{12}\left(p(t)+\frac{\sigma_{2} I_{2}}{2 \pi D_{0}}\right)-\phi_{1} I_{1}, \\
\frac{d I_{2}}{d t} & =\beta_{1} S_{2} \gamma_{21}\left(p(t)+\frac{\sigma_{1} I_{1}}{2 \pi D_{0}}\right)+\beta_{2} S_{2} \gamma_{22}\left(p(t)+\frac{\sigma_{2} I_{2}}{2 \pi D_{0}}\right)-\phi_{2} I_{2}, \\
\frac{d p}{d t} & =-p+\frac{1}{|\Omega|}\left(\sigma_{1} I_{1}+\sigma_{2} I_{2}\right),
\end{aligned}
$$

where $\gamma_{11}$ and $\gamma_{12}$ are the proportions of the residence of patch 1 that are currently in patch 1 and patch 2, respectively, with $\gamma_{11}+\gamma_{12}=1$. Similarly, $\gamma_{21}$ and $\gamma_{22}$ are the proportions of the residence of patch 2 that are currently in patch 1 and patch 2, respectively, with $\gamma_{21}+\gamma_{22}=1$. At the disease free equilibrium (DFE), we construct the Jacobian matrix $F$ for new infections as

$$
F=\left(\frac{\partial \mathcal{F}_{i}}{\partial x_{j}}\right)_{i, j}=\left(\begin{array}{ccc}
\frac{\sigma_{1} \beta_{1} \gamma_{11} N_{1}(0)}{2 \pi D_{0}} & \frac{\sigma_{2} \beta_{2} \gamma_{12} N_{1}(0)}{2 \pi D_{0}} & \left(\beta_{1} \gamma_{11}+\beta_{2} \gamma_{12}\right) N_{1}(0) \\
\frac{\sigma_{1} \beta_{1} \gamma_{21} N_{2}(0)}{2 \pi D_{0}} & \frac{\sigma_{2} \beta_{2} \gamma_{22} N_{2}(0)}{2 \pi D_{0}} & \left(\beta_{1} \gamma_{21}+\beta_{2} \gamma_{22}\right) N_{2}(0) \\
0 & 0 & 0
\end{array}\right),
$$

where the functions $\left(\mathcal{F}_{1}, \mathcal{F}_{2}, \mathcal{F}_{2}\right) \equiv\left(I_{1}^{\prime}, I_{2}^{\prime}, p^{\prime}\right)$ are as given in (3.3) with $\left(x_{1}, x_{2}, x_{3}\right) \equiv\left(I_{1}, I_{2}, p\right)$. Similarly, from (3.3), we construct the Jacobian matrix $V$ for the rates of transfer of individuals between the infected compartments as

$$
V=\left(\frac{\partial \mathcal{V}_{i}}{\partial x_{j}}\right)_{i, j}=\left(\begin{array}{ccc}
\phi_{1} & 0 & 0 \\
0 & \phi_{2} & 0 \\
-\frac{\sigma_{1}}{|\Omega|} & -\frac{\sigma_{2}}{|\Omega|} & 1
\end{array}\right)
$$

Upon finding the inverse of $V$ and multiplying by $F$ (3.4) from the left, we obtain the next generation matrix defined by $\mathcal{M}=F V^{-1}$. Our desired basic reproduction number is the spectral radius of the next generation matrix. Computing the eigenvalues of this matrix, we obtain the basic reproduction number of our model as

$$
\mathcal{R}_{0}=\frac{\mho_{11}+\mho_{22}}{4 \pi \phi_{1} \phi_{2}|\Omega| D_{0}}+\frac{\sqrt{\mho_{11}^{2}+*+\mho_{22}^{2}}}{2 \phi_{1} \phi_{2}},
$$


where $\mho_{11}, \mho_{22}$, and $*$ are defined as follows

$$
\begin{gathered}
\mho_{11}=\left(2 \pi D_{0}\left(\beta_{1} \gamma_{11}+\beta_{2} \gamma_{12}\right)+\beta_{1} \gamma_{11}|\Omega|\right) N_{1}(0) \phi_{2} \sigma_{1}, \\
\mho_{22}=\left(2 \pi D_{0}\left(\beta_{1} \gamma_{21}+\beta_{2} \gamma_{22}\right)+\beta_{2} \gamma_{22}|\Omega|\right) N_{2}(0) \phi_{1} \sigma_{2}, \\
*=2 \phi_{1} \phi_{2} \sigma_{1} \sigma_{2} N_{1}(0) N_{2}(0)\left(\frac{\left(\beta_{1} \gamma_{11}+\beta_{2} \gamma_{12}\right)}{2 \pi D_{0}|\Omega|^{2}} \mho_{22}^{-}-\frac{\beta_{1} \gamma_{11}}{4 \pi^{2} D_{0}^{2}|\Omega|} \mho_{22}+\frac{\beta_{1} \gamma_{21}}{4 \pi^{2} D_{0}^{2}|\Omega|} \mho_{12}^{-}+\frac{\beta_{2} \gamma_{12}}{4 \pi^{2} D_{0}^{2}|\Omega|} \mho_{21}^{-}\right) .
\end{gathered}
$$

Here, the variables $\mho_{22}^{-}, \mho_{12}^{-}$and $\mho_{21}^{-}$are given by

$$
\begin{gathered}
\mho_{22}^{-}=\left(2 \pi D_{0}\left(\beta_{1} \gamma_{21}+\beta_{2} \gamma_{22}\right)-\beta_{2} \gamma_{22}|\Omega|\right), \quad \mho_{12}^{-}=\left(4 \pi D_{0}\left(\beta_{1} \gamma_{11}+\beta_{2} \gamma_{12}\right)+\beta_{2} \gamma_{12}|\Omega|\right), \\
\mho_{21}^{-}=\left(4 \pi D_{0}\left(\beta_{1} \gamma_{21}+\beta_{2} \gamma_{22}\right)+\beta_{1} \gamma_{21}|\Omega|\right),
\end{gathered}
$$

To better understand the basic reproduction number $\mathcal{R}_{0}$ (3.6), we consider some limiting scenarios and make simplifying assumptions on the mixing pattern between the two populations:

1. In the well-mixed limit, where $D_{0} \rightarrow \infty$, the basic reproduction number in (3.6) reduces to

$$
\mathcal{R}_{0}^{\infty}=\left(\beta_{1} \gamma_{11}+\beta_{2} \gamma_{12}\right) \mathcal{R}_{1}+\left(\beta_{1} \gamma_{21}+\beta_{2} \gamma_{22}\right) \mathcal{R}_{2},
$$

where $\mathcal{R}_{1}=N_{1}(0) \sigma_{1} /\left(\phi_{1}|\Omega|\right)$ and $\mathcal{R}_{2}=N_{2}(0) \sigma_{2} /\left(\phi_{2}|\Omega|\right)$. The first term in (3.9) given by $\left(\beta_{1} \gamma_{11}+\beta_{2} \gamma_{12}\right) \mathcal{R}_{1}$ accounts for the secondary infections caused indirectly by a quantity $\sigma_{1}$ of the pathogen shed by a single infected individual in patch 1 per unit time for a time period $1 / \phi_{1}$. A similar explanation holds for the second term in (3.9) given by $\left(\beta_{1} \gamma_{21}+\beta_{2} \gamma_{22}\right) \mathcal{R}_{2}$, but in terms of patch 2 .

2. For a symmetric proportionate mixing pattern, where $\gamma_{11}=\gamma_{21}=\gamma_{1}$, and $\gamma_{12}=\gamma_{22}=\gamma_{2}$, with $\gamma_{1}+\gamma_{2}=1$ and $\gamma_{11} \gamma_{22}-\gamma_{12} \gamma_{21}=0$, the reproduction number in (3.9) becomes

$$
\mathcal{R}_{0}^{\infty}=\frac{\left(\beta_{1} \gamma_{1}+\beta_{2} \gamma_{2}\right) N_{1}(0) \sigma_{1}}{\phi_{1}|\Omega|}+\frac{\left(\beta_{1} \gamma_{1}+\beta_{2} \gamma_{2}\right) N_{2}(0) \sigma_{2}}{\phi_{2}|\Omega|}
$$

3. When there is no mobility, that is, the members of each patch only mix with individuals in their patch, we have $\gamma_{11}=\gamma_{22}=1$ (consequently, $\gamma_{12}=\gamma_{21}=0$ ). Therefore, the reproduction number in (3.9) reduces to

$$
\mathcal{R}_{0}^{\infty}=\frac{\beta_{1} N_{1}(0) \sigma_{1}}{\phi_{1}|\Omega|}+\frac{\beta_{2} N_{2}(0) \sigma_{2}}{\phi_{2}|\Omega|} .
$$

Note that this scenario is the same as that of section 4 of [14], and the reduced reproduction number (3.11) for this scenario is the same as equation 4.9 of [14].

Our numerical simulations will give further explanations of $\mathcal{R}_{0}$ (for the case where $D_{0}=O(1)$ ) and $\mathcal{R}_{0}^{\infty}$ (for the well-mixed limit $D_{0} \rightarrow \infty$ ). We summarize the implications of the reproduction number $\mathcal{R}_{0}^{\infty}$ in the following easily-proved Theorem.

Theorem 1. For the well-mixed limit $D_{0} \rightarrow \infty$ for the system (3.2), the infection dies out whenever $\mathcal{R}_{0}^{\infty}<1$, while an epidemic occurs whenever $\mathcal{R}_{0}^{\infty}>1$.

\subsection{Final size relation}

To represent the epidemic size in terms of the basic reproduction number and the model parameters, we derive a final size relation for the two-patch epidemic model (2.18). Follow the approach used in $[1,4,5,7,8,9,10,11,14,15]$, we obtain

$$
\begin{aligned}
\log \frac{S_{10}}{S_{1 \infty}}= & \left(\beta_{1} \gamma_{11}+\beta_{2} \gamma_{12}\right) p_{0}+\left(\beta_{1} \gamma_{11}+\beta_{2} \gamma_{12}\right) \mathcal{R}_{1}\left\{1-\frac{S_{1 \infty}}{N_{1}(0)}\right\}+\frac{\sigma_{1} N_{1}(0) \beta_{1} \gamma_{11}}{2 \pi D_{0} \phi_{1}}\left\{1-\frac{S_{1 \infty}}{N_{1}(0)}\right\} \\
& +\left(\beta_{1} \gamma_{11}+\beta_{2} \gamma_{12}\right) \mathcal{R}_{2}\left\{1-\frac{S_{2 \infty}}{N_{2}(0)}\right\}+\frac{\sigma_{2} N_{2}(0) \beta_{2} \gamma_{12}}{2 \pi D_{0} \phi_{2}}\left\{1-\frac{S_{2 \infty}}{N_{2}(0)}\right\},
\end{aligned}
$$


and

$$
\begin{aligned}
\log \frac{S_{20}}{S_{2 \infty}}= & \left(\beta_{1} \gamma_{21}+\beta_{2} \gamma_{22}\right) p_{0}+\left(\beta_{1} \gamma_{21}+\beta_{2} \gamma_{22}\right) \mathcal{R}_{1}\left\{1-\frac{S_{1 \infty}}{N_{1}(0)}\right\}+\frac{\sigma_{1} N_{1}(0) \beta_{1} \gamma_{21}}{2 \pi D_{0} \phi_{1}}\left\{1-\frac{S_{1 \infty}}{N_{1}(0)}\right\} \\
& +\left(\beta_{1} \gamma_{21}+\beta_{2} \gamma_{22}\right) \mathcal{R}_{2}\left\{1-\frac{S_{2 \infty}}{N_{2}(0)}\right\}+\frac{\sigma_{2} N_{2}(0) \beta_{2} \gamma_{22}}{2 \pi D_{0} \phi_{2}}\left\{1-\frac{S_{2 \infty}}{N_{2}(0)}\right\},
\end{aligned}
$$

where $\mathcal{R}_{1}$ and $\mathcal{R}_{2}$ are as defined in (3.9), $p_{0}$ is the initial average density of pathogens, $N_{1}(0)$ and $N_{2}(0)$ are the initial populations in patch 1 and patch 2, respectively. Here, $S_{10}$ and $S_{20}$ denote the initial susceptible populations in the patch 1 and 2 respectively, while $S_{1 \infty}$ and $S_{2 \infty}$ are the susceptible populations left in patch 1 and 2 after the outbreak. The remaining parameters are as defined in Table 1.

The final size relations in (3.12a) and (3.12b) for patch 1 and 2, respectively, imply that $S_{1 \infty}>0$ and $S_{2 \infty}>0$. They give the relationship between the basic reproduction number $R_{0}$ and the final epidemic size in patch 1 and 2, respectively. Note that the total number of infected individuals in patch 1 and 2 over the epidemic period are respectively given by $N_{1}(0)-S_{1 \infty}$ and $N_{2}(0)-S_{2 \infty}$, which can be described in terms of the attack rates/ratios as $\left[1-\frac{S_{1 \infty}}{N_{1}(0)}\right]$ and $\left[1-\frac{S_{2 \infty}}{N_{2}(0)}\right]$ as in [5]. The final size relation (3.12) takes a simpler form using the following assumptions.

1. In the case where the outbreak begins with infected individuals and no pathogens, that is, $I_{1}(0) \neq 0, I_{2}(0) \neq 0$ and $p_{0}=0$, the final size relation for patch 1 and 2 in (3.12a) and (3.12b) can be written as

$$
\begin{aligned}
\log \frac{S_{10}}{S_{1 \infty}}= & \left(\beta_{1} \gamma_{11}+\beta_{2} \gamma_{12}\right) \mathcal{R}_{1}\left\{1-\frac{S_{1 \infty}}{N_{1}(0)}\right\}+\frac{\sigma_{1} N_{1}(0) \beta_{1} \gamma_{11}}{2 \pi D_{0} \phi_{1}}\left\{1-\frac{S_{1 \infty}}{N_{1}(0)}\right\} \\
& +\left(\beta_{1} \gamma_{11}+\beta_{2} \gamma_{12}\right) \mathcal{R}_{2}\left\{1-\frac{S_{1 \infty}}{N_{1}(0)}\right\}+\frac{\sigma_{2} N_{2}(0) \beta_{2} \gamma_{12}}{2 \pi D_{0} \phi_{2}}\left\{1-\frac{S_{2 \infty}}{N_{2}(0)}\right\},
\end{aligned}
$$

and

$$
\begin{aligned}
\log \frac{S_{20}}{S_{2 \infty}}= & \left(\beta_{1} \gamma_{21}+\beta_{2} \gamma_{22}\right) \mathcal{R}_{1}\left\{1-\frac{S_{1 \infty}}{N_{1}(0)}\right\}+\frac{\sigma_{1} N_{1}(0) \beta_{1} \gamma_{21}}{2 \pi D_{0} \phi_{1}}\left\{1-\frac{S_{1 \infty}}{N_{1}(0)}\right\} \\
& +\left(\beta_{1} \gamma_{21}+\beta_{2} \gamma_{22}\right) \mathcal{R}_{2}\left\{1-\frac{S_{2 \infty}}{N_{2}(0)}\right\}+\frac{\sigma_{2} N_{2}(0) \beta_{2} \gamma_{22}}{2 \pi D_{0} \phi_{2}}\left\{1-\frac{S_{2 \infty}}{N_{2}(0)}\right\} .
\end{aligned}
$$

2. In the limit $D_{0} \rightarrow \infty$ (well-mixed), the final size relation (3.13) and (3.14) become

$$
\begin{aligned}
& \log \frac{S_{10}}{S_{1 \infty}}=\left(\beta_{1} \gamma_{11}+\beta_{2} \gamma_{12}\right) \mathcal{R}_{1}\left\{1-\frac{S_{1 \infty}}{N_{1}(0)}\right\}+\left(\beta_{1} \gamma_{11}+\beta_{2} \gamma_{12}\right) \mathcal{R}_{2}\left\{1-\frac{S_{2 \infty}}{N_{2}(0)}\right\}, \\
& \log \frac{S_{20}}{S_{2 \infty}}=\left(\beta_{1} \gamma_{21}+\beta_{2} \gamma_{22}\right) \mathcal{R}_{1}\left\{1-\frac{S_{1 \infty}}{N_{1}(0)}\right\}+\left(\beta_{1} \gamma_{21}+\beta_{2} \gamma_{22}\right) \mathcal{R}_{2}\left\{1-\frac{S_{2 \infty}}{N_{2}(0)}\right\} .
\end{aligned}
$$

This result can be written in a matrix form as

$$
\left(\begin{array}{l}
\log \frac{S_{10}}{S_{1 \infty}} \\
\log \frac{S_{20}}{S_{2 \infty}}
\end{array}\right)=\left(\begin{array}{cc}
\mathbb{M}_{11} & \mathbb{M}_{12} \\
\mathbb{M}_{21} & \mathbb{M}_{22}
\end{array}\right)\left(\begin{array}{c}
1-\frac{S_{1 \infty}}{N_{1}(0)} \\
1-\frac{S_{2 \infty}}{N_{2}(0)}
\end{array}\right), \quad \text { where } \quad \mathbb{M}=\left(\begin{array}{ll}
\left(\beta_{1} \gamma_{11}+\beta_{2} \gamma_{12}\right) \mathcal{R}_{1} & \left(\beta_{1} \gamma_{11}+\beta_{2} \gamma_{12}\right) \mathcal{R}_{2} \\
\left(\beta_{1} \gamma_{21}+\beta_{2} \gamma_{22}\right) \mathcal{R}_{1} & \left(\beta_{1} \gamma_{21}+\beta_{2} \gamma_{22}\right) \mathcal{R}_{2}
\end{array}\right) \text {. }
$$

3. If the mixing is proportionate, that is $\gamma_{11}=\gamma_{21}=\gamma_{1}$, and $\gamma_{12}=\gamma_{22}=\gamma_{2}$, the final size relation (3.16) becomes

$$
\left(\begin{array}{c}
\log \frac{S_{10}}{S_{1 \infty}} \\
\log \frac{S_{20}}{S_{2 \infty}}
\end{array}\right)=\left(\begin{array}{cc}
\mathbb{N}_{11} & \mathbb{N}_{12} \\
\mathbb{N}_{21} & \mathbb{N}_{22}
\end{array}\right)\left(\begin{array}{c}
1-\frac{S_{1 \infty}}{N_{1}(0)} \\
1-\frac{S_{2 \infty}}{N_{2}(0)}
\end{array}\right), \quad \text { where } \quad \mathbb{N}=\left(\begin{array}{ll}
\left(\beta_{1} \gamma_{1}+\beta_{2} \gamma_{2}\right) \mathcal{R}_{1} & \left(\beta_{1} \gamma_{1}+\beta_{2} \gamma_{2}\right) \mathcal{R}_{2} \\
\left(\beta_{1} \gamma_{1}+\beta_{2} \gamma_{2}\right) \mathcal{R}_{1} & \left(\beta_{1} \gamma_{1}+\beta_{2} \gamma_{2}\right) \mathcal{R}_{2}
\end{array}\right) .
$$


4. If the mixing is like-with-like (no mobility), that is $\gamma_{11}=\gamma_{22}=1$, and $\gamma_{12}=\gamma_{21}=0$, the final size relation (3.16) reduces to

$$
\left(\begin{array}{l}
\log \frac{S_{10}}{S_{1 \infty}} \\
\log \frac{S_{20}}{S_{2 \infty}}
\end{array}\right)=\left(\begin{array}{ll}
\mathbb{W}_{11} & \mathbb{W}_{12} \\
\mathbb{W}_{21} & \mathbb{W}_{22}
\end{array}\right)\left(\begin{array}{c}
1-\frac{S_{1 \infty}}{N_{1}(0)} \\
1-\frac{S_{2 \infty}}{N_{2}(0)}
\end{array}\right), \quad \text { where } \quad \mathbb{W}=\left(\begin{array}{ll}
\beta_{1} \mathcal{R}_{1} & \beta_{1} \mathcal{R}_{2} \\
\beta_{2} \mathcal{R}_{1} & \beta_{2} \mathcal{R}_{2}
\end{array}\right)
$$

which can be written as

$$
\beta_{2} \log \frac{S_{10}}{S_{1 \infty}}=\beta_{1} \log \frac{S_{20}}{S_{2 \infty}} .
$$

We can further reduce (3.19) to obtain

$$
\left[\frac{S_{10}}{S_{1 \infty}}\right]^{\beta_{2}}=\left[\frac{S_{20}}{S_{2 \infty}}\right]^{\beta_{1}}
$$

If $\beta_{1}>\beta_{2}$ in (3.20), then

$$
1-\log \frac{S_{10}}{S_{1 \infty}}>1-\log \frac{S_{20}}{S_{2 \infty}},
$$

which implies that the attack rate/ratio is greater in the more active patch.

The assumptions above and their effects on the epidemic peak and time will be explored and discussed in the numerical simulations.

\subsection{Numerical simulations}

We present numerical simulations of the dimensional coupled PDE-ODE model (3.1) and the reduced system of ODEs (3.2) for two population patches. For all scenarios considered in this section, these patches are located at $\boldsymbol{x}_{1}=(0.5,0)$ and $\boldsymbol{x}_{2}=(-0.5,0)$ for patch 1 and 2, respectively, and the coupled PDE-ODE model is solved using FlexPDE6 [20]. We aim to study the effect of pathogen diffusion and human mobility on the disease dynamics in an heterogeneous mixing environment.

In Figure 1, we used the reduced ODE system (3.2) to study the effect of the diffusion rate of pathogens and heterogeneous mixing between two populations on the transmission dynamics of the diseases in the populations. For the results in the left panel (initial conditions: $S_{1}(0)=299 / 300, I_{1}(0)=1 / 300, R_{1}(0)=0, S_{2}(0)=249 / 250$, $I_{2}(0)=1 / 250, R_{2}(0)=0$, and $p(0)=0$ ), we have only infected individuals with no pathogens at the beginning of the outbreak, while for those in the right panel (initial conditions: $S_{1}(0)=300, I_{1}(0)=0, R_{1}(0)=0, S_{2}(0)=250, I_{2}(0)=$ $0, R_{2}(0)=0$, and $\left.p(0)=1\right)$, we have only diffusing pathogens with no infected individuals at the beginning of the epidemic. The second scenario, where the outbreak begins with only pathogens, can be related to a scenario where pathogens diffuse out of an infected population to a completely susceptible population. The solid and dashed curves are for patch 1 and patch 2, respectively. The black, blue, and red curves are for the scaled diffusion rates $D_{0}=0.128$, $D_{0}=2.556$, and $D_{0}=76.687$, respectively. Using the relation for $D_{0}$ and $D$ given in (2.9), these scaled diffusion rates correspond to $D=0.5, D=10$, and $D=300$, respectively.

We observe from the results in the top panel of Figure 1 for the scenario with no human mobility $\left(\gamma_{11}=1, \gamma_{12}=\right.$ $0, \gamma_{21}=0$, and $\left.\gamma_{22}=1\right)$ that, the epidemic peak decreases and the peak time increases, with increase in the scaled diffusion rate. Overall and in this scenario, patch 2 (dashed curves) experiences a higher peak and shorter peak time for all values of $D_{0}$ when compared to patch 1 (solid curves) due to higher transmission and shedding rates in patch 2 (See Table 1 for more details). In addition, the epidemic take-off seems delayed when the outbreak begins with some infectives and no pathogen (left panel), compared to when the outbreak begins with zero infectives and a pathogen (right panel). This observation is more apparent when the diffusion rate is increases (blue and red curves). Similar results obtained using the full coupled PDE-ODE model (3.1) are given in the top panel of Figure 2. In the middle panel of Figure 1, we have the results for the case of symmetric proportional mixing $\left(\gamma_{11}=\gamma_{12}=0.5\right.$ and $\left.\gamma_{21}=\gamma_{22}=0.5\right)$. For this scenario, the activity level in the two populations are assumed to be the same and as a result, an equal proportion of the two population are mixing at all time. As observed from the results in the middle 


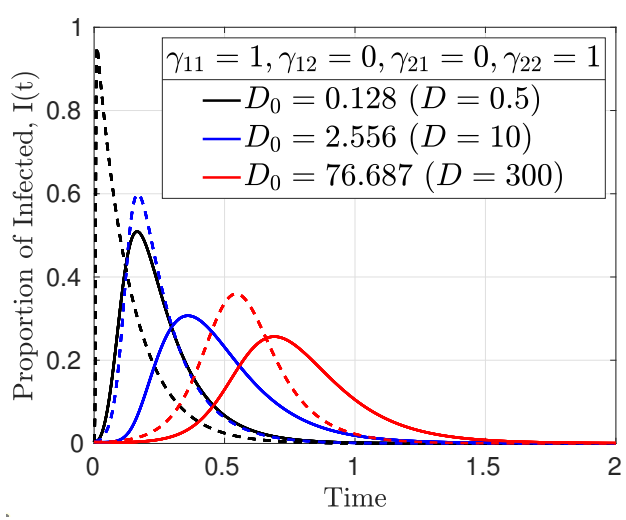

(a) No human mobility

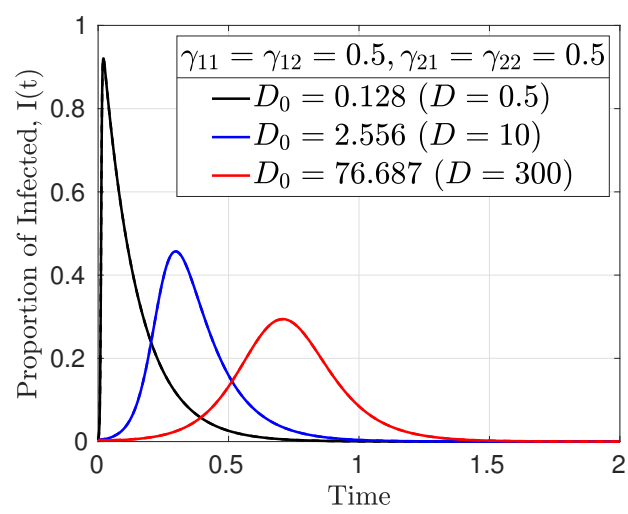

(c) Symmetric proportionate mixing

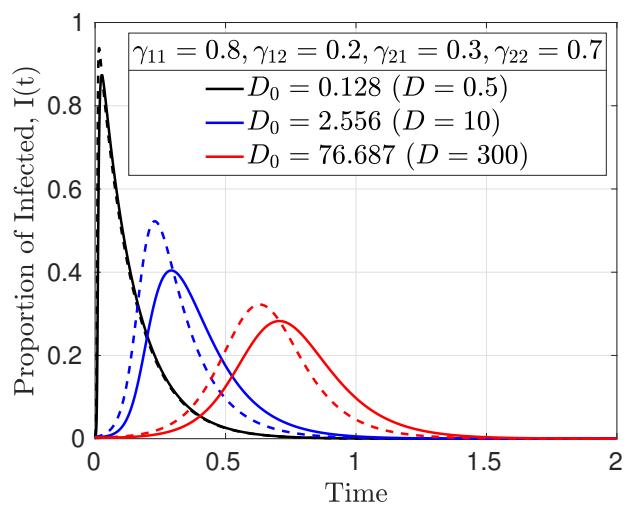

(e) Low human mobility

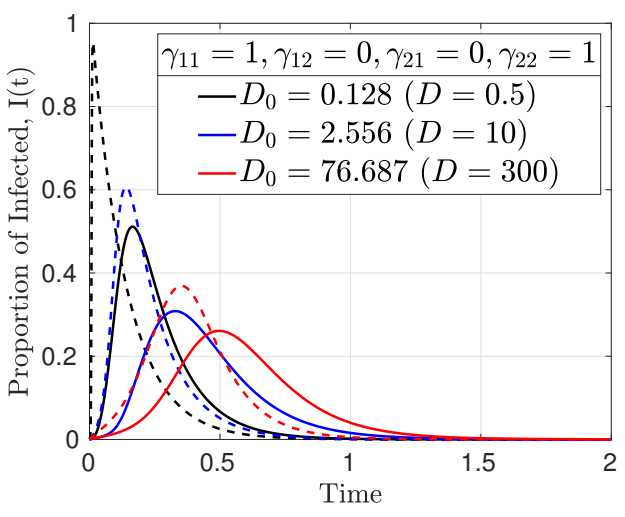

(b) No human mobility

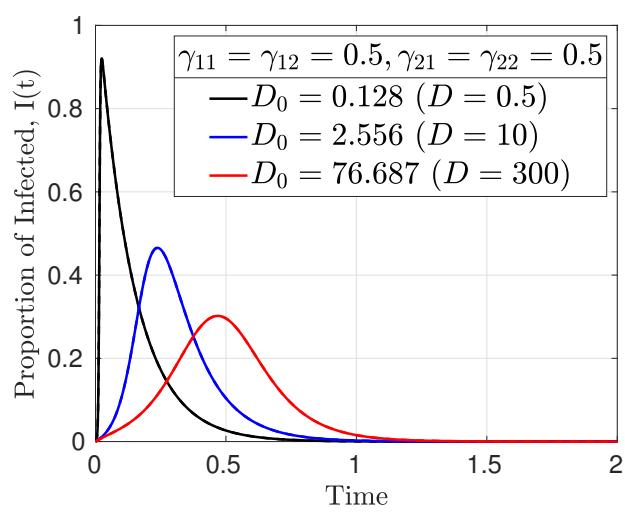

(d) Symmetric proportionate mixing

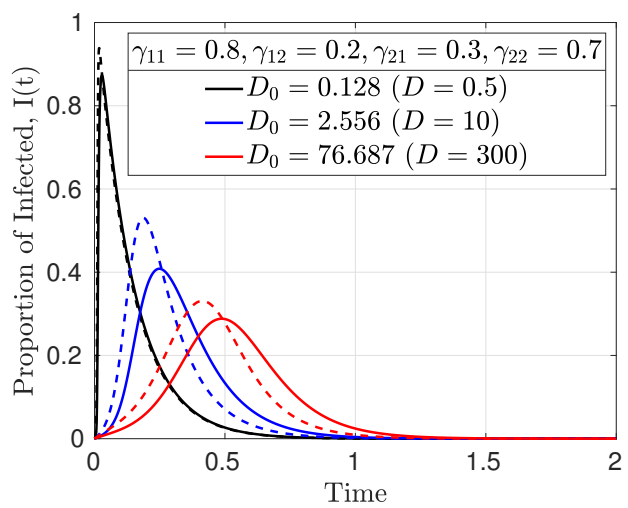

(f) Low human mobility

Figure 1: Effect of pathogen diffusion and human mobility on disease dynamics (ODE model). Numerical simulations of the ODE system (3.2) for different diffusion rate of pathogens and human mobility. Left panel: infection starts with no pathogen, with initial conditions $S_{1}(0)=299 / 300$, $I_{1}(0)=1 / 300, R_{1}(0)=0, S_{2}(0)=249 / 250, I_{2}(0)=1 / 250, R_{2}(0)=0$, and $p(0)=0$, and right panel: infection starts with only pathogens with initial conditions $S_{1}(0)=300, I_{1}(0)=0, R_{1}(0)=0, S_{2}(0)=$ $250, I_{2}(0)=0, R_{2}(0)=0$, and $p(0)=1$. Top panel: no human mobility $\left(\gamma_{11}=1, \gamma_{12}=0, \gamma_{21}=0\right.$, and $\left.\gamma_{22}=1\right)$, middle panel: symmetric proportionate mixing $\left(\gamma_{11}=\gamma_{12}=0.5\right.$ and $\left.\gamma_{21}=\gamma_{22}=0.5\right)$, and bottom panel: non-symmetric proportionate mixing (low mobility: $\gamma_{11}=0.8, \gamma_{12}=0.2$ and $\gamma_{21}=0.3, \gamma_{22}=0.7$ ). All other parameters are as given in Table 1. Solid curves are for patch 1 while the dashed curves are for patch 2 .

panel of Figure 1, this mixing patterns lead to a well-mixed (homogeneous) system. Even though the transmission and shedding rates of patch 2 are higher than those of patch 1, our numerical simulations predict identical epidemics 
for the two populations.

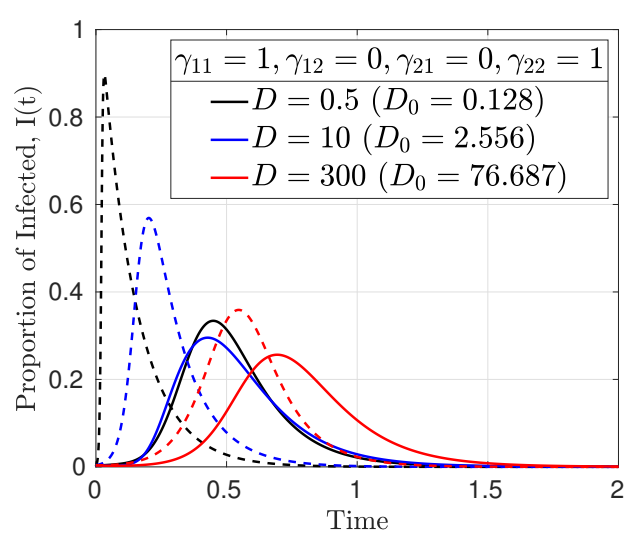

(a) No human mobility

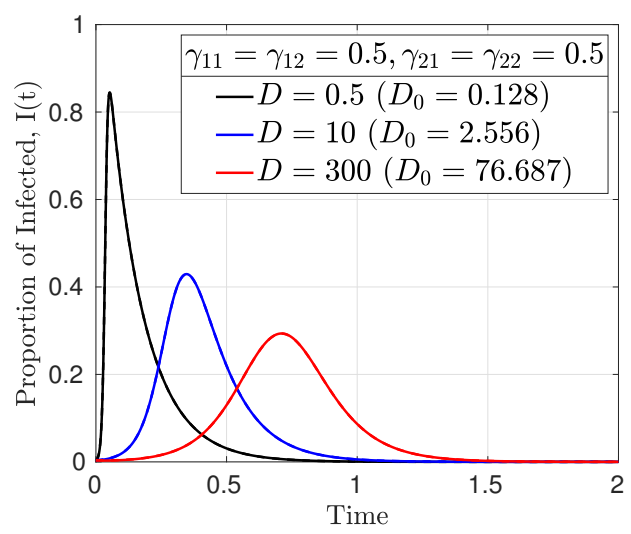

(c) Symmetric proportionate mixing

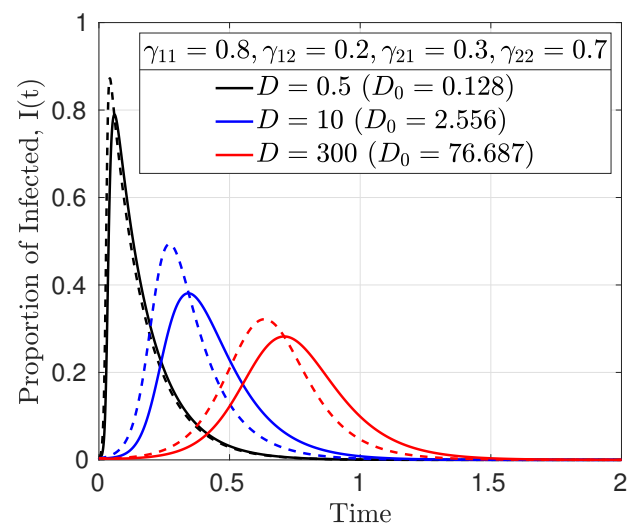

(e) Low human mobility

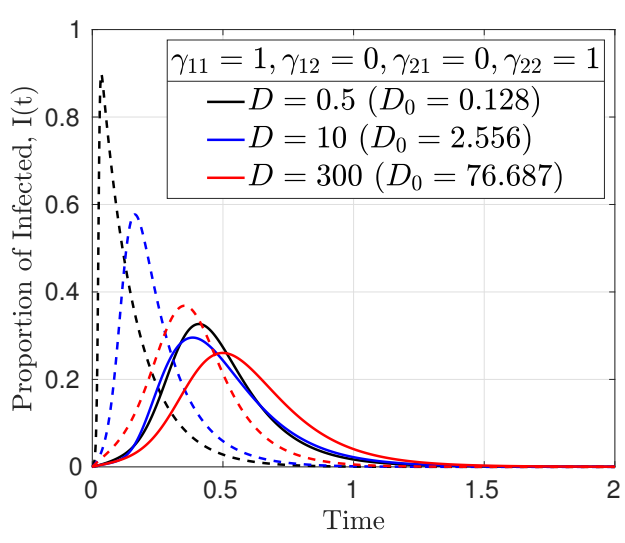

(b) No human mobility

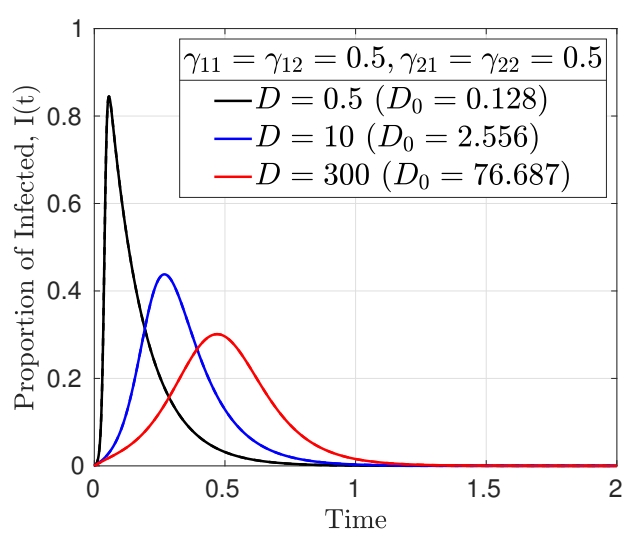

(d) Symmetric proportionate mixing

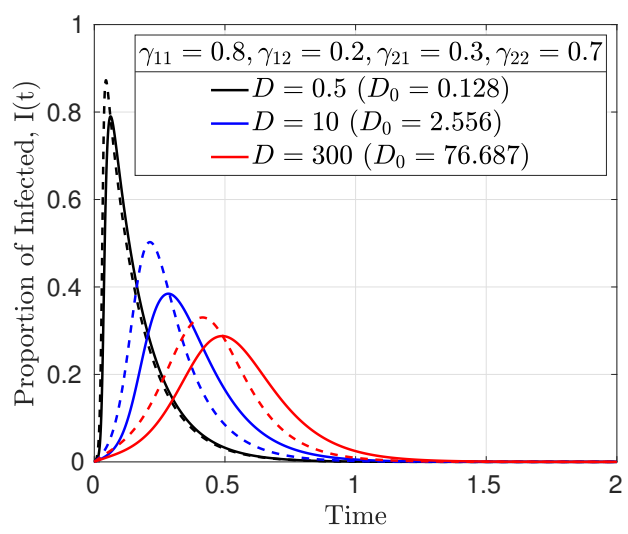

(f) Low human mobility

Figure 2: Effect of pathogen diffusion and human mobility on disease dynamics (PDE-ODE model). Same caption as Figure 1 but for the full PDE-ODE model (3.2).

In the bottom panel of Figure 1, we consider the case of non-symmetric proportionate mixing (low mobility). For this example, we assume that $80 \%$ of the residents of patch 1 mix with only the individuals in their patch, while the remaining $20 \%$ mix with residents of patch $2\left(\gamma_{11}=0.8, \gamma_{12}=0.2\right)$. On the other hand, we assume $70 \%$ of the residents of patch 2 mix with only members of their patch, while the remaining $30 \%$ mix with residents of patch 1 $\left(\gamma_{21}=0.3, \gamma_{22}=0.7\right)$. We observe from the results for this scenario (bottom panel of Figure 1) that the epidemic peak is higher in patch 2 (dashed curves) compared to patch 1 (solid curves) due to higher transmission and shedding rates in patch 2. In this scenario, we say that the residence of patch 2 are having more activities than those in patch 1 since a larger fraction of them is mixing with the individuals in patch 1 than those of patch 1 are mixing with them. 
Comparing the results in the top (no mobility) and bottom (low mobility) panels of Figure 1, we notice that there is an increase in the epidemic peaks for patch 1 when there is mobility compared to when there is no mobility (top panel). These differences in the epidemic size between the two scenarios are due to the heterogeneous mixing between the two populations. On the other hand, there is a slight decrease in the epidemic peaks for patch 2 when there is mobility (bottom panel) compared to when there is no mobility (top panel). For all the scenarios considered in Figure 1, similar results computed using the full PDE model (3.1) are presented in Figure 2. Both results agree well.

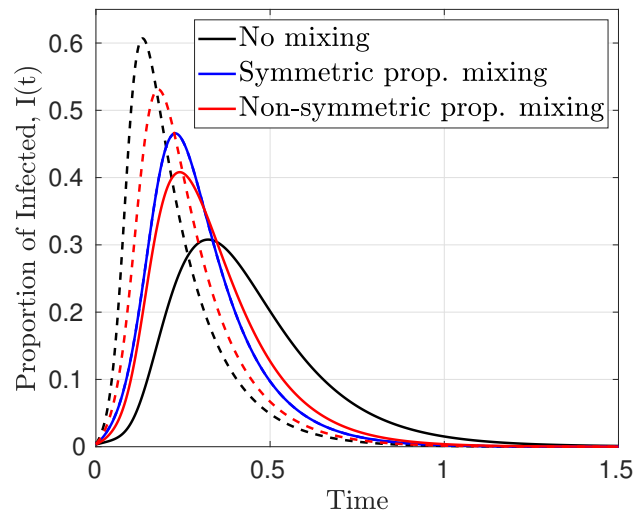

(a) Reduced ODE model (3.2)

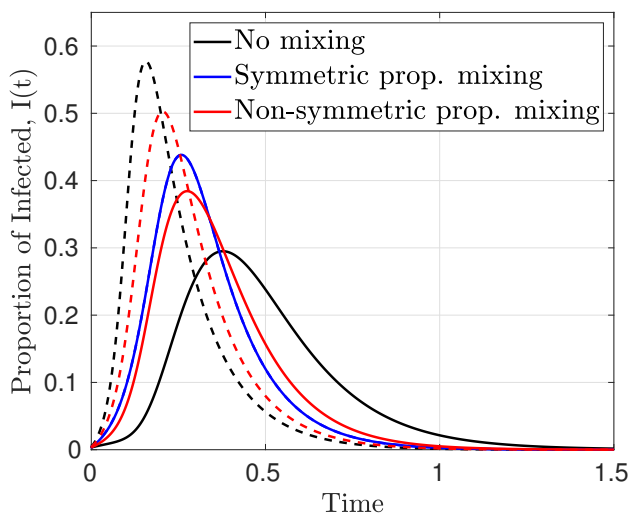

(b) Coupled ODE-PDE model (3.1)

Figure 3: Effect of population mixing patterns on disease dynamics. Numerical simulations of the ODE system (3.2) (left panel) and the coupled PDE-ODE model (3.1) (right panel) for two population patches and different population mixing patterns. The solid and dashed curves are for patch 1 and patch 2, respectively. The black, blue and red curves are respectively for the scenarios with no mobility $\left(\gamma_{11}=1, \gamma_{12}=0, \gamma_{21}=0\right.$, and $\left.\gamma_{22}=1\right)$, symmetric mixing $\left(\gamma_{11}=\gamma_{12}=0.5\right.$ and $\left.\gamma_{21}=\gamma_{22}=0.5\right)$ and non-symmetric proportionate mixing (low mobility: $\gamma_{11}=0.8, \gamma_{12}=0.2$ and $\gamma_{21}=0.3, \gamma_{22}=0.7$ ). The initial conditions used are $S_{1}(0)=299 / 300, I_{1}(0)=1 / 300, R_{1}(0)=0, S_{2}(0)=249 / 250, I_{2}(0)=1 / 250, R_{2}(0)=0$, and $p(0)=1$. For the PDE-ODE model, we used $D=10$ (right panel), corresponding to $D_{0}=2.556$ for the reduce ODE system (left panel). All other parameters are as given in Table 1.

The results in Figure 3 are used to study the effect of heterogeneous mixing on the disease dynamics for a fixed diffusion rate of pathogens, $D=10$ (corresponding to $D_{0}=2.556$ ). The initial conditions used are $S_{1}(0)=299 / 300$, $I_{1}(0)=1 / 300, R_{1}(0)=0, S_{2}(0)=249 / 250, I_{2}(0)=1 / 250, R_{2}(0)=0$, and $p(0)=1$, with other parameters as given in Table 1. Similar to the results presented in Figures 1 and 2, the solid and dashed curves are for patch 1 and patch 2 respectively. The results in the left panel were obtained using the reduced ODE system (3.2) and those in right panel were obtained using the coupled PDE-ODE model (3.1). The black curves show the results for when there is no movement between the two populations, the blue curves are for symmetric proportionate mixing, where half of the two populations are mixing, while the red curves are for non-symmetric proportionate mixing (low mobility). We observe from these results that the no mixing scenario predicts the most difference in the epidemics of the two populations. When there is a symmetric proportionate mixing, an identical epidemic is predicted for the two populations, irrespective of the difference in their transmission and shedding rates. Lastly, for the case of non-symmetric mixing, there is a decrease in the epidemic for the population with higher shedding and transmission rates (patch 1) and an increase in the epidemic for the other population with lower transmission and shedding rates (patch 2). Comparing the results in the left and right panel of Figure 3, we notice that the results from the reduced system of ODEs (3.2) agree well with those obtained using the full coupled PDE-ODE model (3.1). This shows that the reduced ODE system provides a good approximation for the coupled PDE-ODE system.

\section{Discussion}

We have extended the novel coupled PDE-ODE model developed in [14] for studying the transmission dynamics of airborne diseases to include human mobility. Human mobility between populations was incorporated using the 
Lagrangian approach. This model is used to study the indirect transmission of airborne diseases, where a susceptible individual becomes infected after coming in contact with pathogens. Infected individuals shed pathogens into the environment at some rate, and the pathogens diffuse and decay in the environment at constant rates. In the limit of fast diffusing pathogens, matched asymptotic analysis was used to reduce the PDE-ODE model to a nonlinear system of ODEs for the average density of pathogens in the environment. The reduced ODEs system was then used to derive the basic reproduction number and a final size relation for the epidemic. Numerically simulations of both the coupled PDE-ODE model and the reduced system of ODEs were used to study the effect of the diffusion rate of pathogens and heterogeneous mixing on the dynamics of airborne diseases.

To study the effect of heterogeneous mixing between populations, we considered two non-identical population patches centered at $(0.5,0)$ and $(-0.5,0)$ for patches 1 and 2 , respectively. The differences between the two patches were introduced through the transmission and shedding rates, where patch 2 has higher transmission and shedding rates relative to patch 1 . In addition, we considered three mobility scenarios: no mobility, symmetric proportionate mixing (half populations moving), and non-symmetric proportionate mixing (low mobility). Our simulations results show that even though the two populations are non-identical, symmetric mixing pattern leads to a well-mixed population with identical epidemics in the two populations. This is due to the fact that $50 \%$ of the members of patch 2 (with higher transmission and shedding rates) are mixing with the individuals in patch 1 and vise versa. For non-symmetric proportionate mixing, we assumed that $80 \%$ of the individuals in patch 1 are having contacts with only those in patch 1 , while the remaining $20 \%$ of the population are having their contacts with those in patch 2 . On the other hand, $70 \%$ of the population of patch 2 are having their contact with only those in patch 2, while the remaining 30\% are having their contact with only those in patch 1 . For this mobility pattern, our numerical simulations show an increase in the epidemic for patch 1 (with smaller shedding and transmission rates) compared to when there is no movement. We believe that this increase in the epidemic is due to the contacts made with the individuals in patch 2 by $20 \%$ of those in patch 1, since patch 2 has higher transmission and shedding rates. These contacts would not have occurred if there was no movement. Similarly, the epidemic in patch 2 is noticed to decrease for non-symmetric mixing compared to when there is no movement. This is also due to the contacts made by the $30 \%$ of individuals in patch 2 that are mixing with only those in patch 1 . Overall, our results show that movement between human populations may lead to an increase or decrease in the epidemic size depending on the outbreak situation and infection rate. In addition, they show that movement maybe allowed from the population with high transmission and shedding rate to the other populations with lower rates, but not vice versa.

To study the impact of the diffusing pathogens on the epidemic, we consider two scenarios: when an outbreak starts with infected individuals only and when the outbreak starts with only diffusing pathogens. The latter scenario can be seen as a scenario where pathogens diffuse to completely susceptible populations from infectious populations. Our numerical simulations show that the epidemic takes off faster when the outbreak begins with diffusing pathogens compared to when it begins with infected individuals. Epidemic take-off seems delayed when the outbreak begins with infected individuals because it will take some time for them to shed enough pathogens that will further infect others. We also explore how the diffusion rate of pathogens impact the disease dynamics. We solved the reduced system of ODEs and the coupled PDE-ODE model with small, moderate, and high diffusion rates of pathogens. Our results show that an increase in the diffusion rate of pathogens leads to a lower epidemic peak, and an increase in the epidemic time. Having a high diffusion rate, which may be interpreted as having a windy situation that blows the pathogens around in the air randomly, may lead to the pathogens being blown away from the settlements by the wind, there by leading to a decrease in cases. Even though the analysis used to obtain our reduce system of ODEs is only valid in the limit of fast diffusing pathogens, we observe from our numerical simulations that the results obtained using the ODE model agree well with those of the full coupled PDE-ODE model, even for small diffusion rates of pathogens. This suggests that the reduced ODE system can be used to study the disease dynamics instead of the more complicated PDE-ODE model. An important and unique feature of the ODE system is that it include a diffusion parameter, which can be used to study the effect of diffusion of pathogen on the disease dynamics.

Summarily, the numerical simulations for both the coupled model and the reduced system of ODEs predict a change in the epidemic peak size and time with human mobility and diffusion. Our results show that there is a decrease in the epidemic peak and an increase in the epidemic time as the diffusion rate of pathogens increases in both the coupled PDE-ODE model and the reduced system of ODEs. In the absence of movement, the epidemic is higher in the patch with higher transmission and shedding rates. In addition, when infections start with no pathogens in the air, the model predicts a delay in the epidemic take-off time relative to when infections start with pathogens, and this delay increases 
as the diffusion rate of pathogens increases. Our primary results show that accounting for human mobility in an heterogeneous environment is a great way to account for diseases spread in this heterogeneous world, and it is indeed worth taking into account. We have a similar result with [14] when there is no human mobility between patches, and [2], where they considered only direct transmission pathway.

There are several future directions to the modeling framework presented in this paper. An interesting extension of this work is to allow for infections to take place in the bulk region. In this paper, we assume that infections can only take place when individuals are in the patches. It would also be worthwhile to extend the model to also include direct transmission of infections from host-host. We have considered only the leading-order terms for the reduced ODE system in this study. It would be interesting to include the $O(v)$ terms that incorporate the effect of the location of the patches into the ODE systems. In this instance, the basic reproduction number and final size relation derived from the ODE system will depend on the location of the patches. It is straightforward to extend our model to an n-patch model to study the impact of human mobility on the disease prevalence among multiple regions. Another interesting future direction is to include interventions such as vaccination and treatment, especially in an extreme mobility scenario. A limitation of our study includes the inability to explicitly model the diffusion of pathogens in the population patches. We know in reality that the pathogens would need to diffuse into the population before infections can take place. Another limitation of the model involves not allowing infections to occur outside the population patches (in the bulk region). In this case, the model will become a reaction-diffusion system. Despite these limitations, our modeling framework provides a lot of insight into the potential impact of human mobility on the dynamics of airborne diseases. Our results give insights on boarder control (closing and opening of boarders) between two regions during a pandemic like that of COVID-19. This study serves as a foundation for other studies and its framework can be applied to modeling other infectious diseases.

\section{References}

[1] Julien Arino, Fred Brauer, P Van Den Driessche, James Watmough, and Jianhong Wu. A final size relation for epidemic models. Mathematical Biosciences and Engineering, 4(2):159, 2007.

[2] Derdei Bichara, Yun Kang, Carlos Castillo-Chavez, Richard Horan, and Charles Perrings. Sis and sir epidemic models under virtual dispersal. Bulletin of mathematical biology, 77(11):2004-2034, 2015.

[3] Stephanie A Boone and Charles P Gerba. Significance of fomites in the spread of respiratory and enteric viral disease. Applied and environmental microbiology, 73(6):1687-1696, 2007.

[4] Fred Brauer. Age-of-infection and the final size relation. Math. Biosci. Eng, 5(4):681-690, 2008.

[5] Fred Brauer. Epidemic models with heterogeneous mixing and treatment. Bulletin of mathematical biology, 70(7):1869, 2008.

[6] FRED Brauer. Heterogeneous mixing in epidemic models. Can Appl Math Q, 20(1):1-13, 2012.

[7] Fred Brauer. A final size relation for epidemic models of vector-transmitted diseases. Infectious Disease Modelling, 2(1):12-20, 2017.

[8] Fred Brauer. A new epidemic model with indirect transmission. Journal of biological dynamics, 11(sup2):285-293, 2017.

[9] Fred Brauer. The final size of a serious epidemic. Bulletin of mathematical biology, 81(3):869-877, 2019.

[10] Fred Brauer and Carlos Castillo-Chaavez. Mathematical models for communicable diseases, volume 84. SIAM, 2012.

[11] Fred Brauer, Carlos Castillo-Chavez, and Zhilan Feng. Mathematical models in epidemiology, 2018.

[12] Fred Brauer, Carlos Castillo-Chavez, and Zhilan Feng. Mathematical models in epidemiology, volume 32. Springer, 2019. 
[13] Carlos Castillo-Chavez, Derdei Bichara, and Benjamin R Morin. Perspectives on the role of mobility, behavior, and time scales in the spread of diseases. Proceedings of the National Academy of Sciences, 113(51):14582-14588, 2016.

[14] Jummy F David, Sarafa A Iyaniwura, Michael J Ward, and Fred Brauer. A novel approach to modelling the spatial spread of airborne diseases: an epidemic model with indirect transmission. Mathematical Biosciences and Engineering, 17(4):3294-3328, 2020.

[15] Jummy Funke David. Epidemic models with heterogeneous mixing and indirect transmission. Journal of biological dynamics, 12(1):375-399, 2018.

[16] Odo Diekmann, Johan Andre Peter Heesterbeek, and Johan AJ Metz. On the definition and the computation of the basic reproduction ratio $\mathrm{r} 0$ in models for infectious diseases in heterogeneous populations. Journal of mathematical biology, 28(4):365-382, 1990.

[17] Baltazar Espinoza, Victor Moreno, Derdei Bichara, and Carlos Castillo-Chavez. Assessing the efficiency of movement restriction as a control strategy of ebola. In Mathematical and Statistical Modeling for Emerging and Re-emerging Infectious Diseases, pages 123-145. Springer, 2016.

[18] Trisha Greenhalgh, Jose L Jimenez, Kimberly A Prather, Zeynep Tufekci, David Fisman, and Robert Schooley. Ten scientific reasons in support of airborne transmission of sars-cov-2. The lancet, 397(10285):1603-1605, 2021.

[19] James Holland Jones. Notes on r0. Califonia: Department of Anthropological Sciences, 2007.

[20] PDE solutions Inc. FlexPDE 6, 2019.

[21] Pauline Van den Driessche and James Watmough. Reproduction numbers and sub-threshold endemic equilibria for compartmental models of disease transmission. Mathematical biosciences, 180(1-2):29-48, 2002.

[22] Liang Zhang, Zhi-Cheng Wang, and Yan Zhang. Dynamics of a reaction-diffusion waterborne pathogen model with direct and indirect transmission. Computers $\mathcal{F}$ Mathematics with Applications, 72(1):202-215, 2016. 